\title{
Papel de la biopsia dinámica del ganglio centinela en el cáncer de próstata organoconfinado
}

\author{
Janetschek G*, Jeschke S*, Leeb $\mathrm{K}^{*}$, Prammer P*, Ziegerhofer J**, Sega $\mathrm{W}^{* * *}$. \\ *Servicio de Urología, ${ }^{* *}$ Servicio de Medicina Nuclear, Elisabethinen Hospital, Linz. \\ ***:Servicio de Anatomía Patológica, Barmherzige Schwestern Hospital, Linz, Austria.
}

Actas Urol Esp. 2007;31(6):686-692

\section{RESUMEN}

PAPEL DE LA BIOPSIA DINÁMICA DEL GANGLIO CENTINELA EN EL CÁNCER DE PRÓSTATA ORGANOCONFINADO

La correcta valoración de la afectación ganglionar es de gran importancia en el manejo de los nuevos casos de cáncer de próstata. El estadio precoz de la enfermedad metastásica se manifiesta como pequeños focos microscópicos más que como ganglios linfáticos engrosados. Sin embargo, hasta ahora no hay ninguna técnica de imagen que permita detectar metástasis cuyo diámetro alcance unos pocos milímetros. Por tanto, la linfadenectomía pélvica (LNDP) es el único método fiable para el estadiaje del cáncer de próstata organoconfinado (CPO).

El pilar de la cirugía prostática radioguiada es el uso de un radiofármaco, una molécula transportadora marcada con radionúclido. Tras la inyección en la glándula prostática, el radiofármaco alcanza el territorio linfático y migra al primer escalón linfático, el ganglio centinela (GC). Fuimos los primeros en demostrar que la LDNP del GC se podía llevar a cabo mediante abordaje laparoscópico previo a la prostatectomía radical (PTR) laparoscópica. En nuestra publicación más reciente se presentan los datos de 140 pacientes diagnosticados de CPO entre noviembre de 2001 a enero 2005 en los que se realizó LDNP laparoscópica del GC previa a la PTR, también laparoscópica ${ }^{14}$. En la gammagrafía preoperatoria, se detectaron GC de forma bilateral en 113 pacientes (80,7\%), de forma unilateral en $20(14,2 \%)$ y no se detectaron en 6 $(4,2 \%)$, mientras que en la realizada durante la cirugia se detectaron en 96 pacientes $(68,6 \%), 36(25,7 \%), 8(5,7 \%)$ respectivamente. Los GC se hallaron en la misma localización con ambos procedimientos en 99 de 140 pacientes (70,7\%). $\mathrm{Al}$ menos se objetivó un GC en 133 pacientes (95,3\%).

Cuando la LDNP está indicada, ésta no debería restringirse a la exéresis de adenopatías, según la técnica de LDNP limitada o estándar, sino que tiene que realizarse siguiendo el patrón de la LDNP extendida. Hay poca experiencia con la LDNP del GC, pero todos los datos publicados hasta ahora indican que este procedimiento puede ser una alternativa a la LDNP extendida, ya que permite reducir la extensión de la LDNP sin comprometer la exactitud diagnóstica.

Palabras clave: Ganglio centinela. Linfadenectomía pélvica. Cáncer de próstata organoconfinado.

\section{ABSTRACT}

SENTINEL LYMPH NODE DISSECTION FOR LOCALIZED PROSTATE CANCER

Proper assessment of lymph node status is of crucial importance in the management of newly diagnosed prostate cancer. Early stage metastatic disease takes the form of microscopic tumor-cell deposits rather than grossly enlarged nodes. So far there is no imaging technique, however, which allows detecting small metastases in the range of a few millimetres. Therefore pelvic lymph node dissection (PLND) is the only reliable method of staging for clinically localized prostate cancer. The cornerstone of radioguided prostate surgery is a radiopharmaceutical - a carrier molecule labeled by radionuclide. After injection to at the prostate, the radiopharmaceutical crosses the lymphatic pores and migrates into the lymph vessels and from there to the first echelon of lymph nodes. We were the first to show that sentinel PLND can be performed by means of laparoscopy preceding laparoscopic radical prostatectomy. Our most recent publication presents data of 140 patients with clinically localized prostate cancer in which laparoscopic sentinel PLND was performed preceding radical prostatectomy from November 2001 to January $2005^{14}$. On the preoperative scintigraphy SLNs were detected bilaterally,unilaterally, not on the pelvic-walls in $113(80.7 \%), 20(14.2 \%)$ and $6(4.2 \%)$ patients and intraoperatively in 96 (68.6\%), $36(25.7 \%), 8(5.7 \%)$ patients respectively. In 99 out of 140 patients $(70.7 \%)$ intraoperatively SLN was detected in the same position as on preoperative scan. At least one SLN was detected in 133 patients (95.3\%).

Whenever PLND is indicated it should not be limited to lymph node sampling as provided by standard limited PLND but has to be performed in the template of extended PLND. There is only limited experience with sentinel PLND, but all the data collected so far indicate that this method has the potential to become an alternative to extended PLND since it allows for reduction of the extent of PLND without compromising diagnostic accuracy. 
$L^{2}$ a correcta valoración de la afectación ganglionar es de gran importancia en el manejo de los nuevos casos de cáncer de próstata. El estadio precoz de la enfermedad metastásica se manifiesta como pequeños focos microscópicos más que como ganglios linfáticos engrosados. Sin embargo, hasta ahora no hay ninguna técnica de imagen que permita detectar metástasis cuyo diámetro alcance unos pocos milímetros. Por tanto, la linfadenectomía pélvica (LNDP) es el único método fiable para el estadiaje del cáncer de próstata organoconfinado (CPO). Aunque la LDNP es una técnica establecida en el estadiaje del CP, ciertos aspectos de dicho procedimiento, como son su indicación y la extensión de la misma, están todavía sujetos a cierta controversia.

A pesar de que se sabe desde hace mucho tiempo que la LDNP estándar sólo engloba los ganglios que albergan la fosa obturatriz, en la práctica se sigue ignorando que la afectación linfática primaria pueda ocurrir en otras localizaciones. Cuando la literatura actual estima la incidencia de adenopatías positivas entre el 2-7\%, se basan en trabajos en los que se ha utilizado LDNP limitada. Como consecuencia de lo mencionado anteriormente, los estudios y nomogramas basados en este tipo de LDN tienden a infravalorar la incidencia real de enfermedad avanzada, por lo que inducen a conclusiones erróneas respecto a la exactitud del estadiaje y manejo terapéutico. Influido por esta relativa baja tasa de ganglios positivos en CPO, se ha cuestionado la necesidad de realizar LDNP rutinaria en aquellos pacientes que se vayan a someter a una PTR.

Estudios gammagráficos han demostrado que el territorio linfático de la glándula prostática drena en 3 sistemas, siendo el más importante aquel que drena en los ganglios iliacos internos, que habitualmente no se engloba en la LNDP ${ }^{1}$. De manera no poco frecuente, se han aislado adenopatías únicas alrededor de los vasos iliacos internos y externos. Studer y su grupo fueron los primeros en demostrar de forma clara que la tasa de detección de pequeñas metástasis ganglionares aisladas aumentaba de forma considerable cuando se realizaba una LDNP extendida, y que si se realizaba exclusivamente la LDNP de la fosa obturatriz, casi la mitad de estas metástasis no hubieran sido detectadas ${ }^{2}$.
La LDNP extendida comparada con la limitada, es una intervención más prolongada que conlleva una morbilidad incrementada inherente a la técnica quirúrgica. Con el fin de simplificar la disección de la LDNP extendida y reducir así esta morbilidad, sin tener que comprometer la fiabilidad diagnóstica, surgió el concepto de la biopsia del ganglio centinela, cuya utilidad ya se había probado en el manejo del melanoma, cáncer de mama y testículo, siendo exitosamente experimentada junto la LDNP por Wawroschek ${ }^{3}$. Nosotros fuimos los primeros en demostrar que la LND del GC puede realizarse a través de un abordaje laparoscópico previo a la $\mathrm{PTR}^{4}$.

\section{CONCEPTO Y TÉCNICA DE LA LDNP DEL GC}

El pilar de la cirugía prostática radioguiada es el uso de un radiofármaco, una molécula transportadora marcada con radionúclido. Tras la inyección en la glándula prostática, el radiofármaco alcanza el territorio linfático y migra al primer escalón linfático, el GC. Aquí experimenta una pinocitosis y se acumula en los reticulocitos. La señal generada por el radiofármaco se detectará en ese momento mediante gammagrafia extracorpórea y escáner intraoperatorio (Figs. 1 y 2).

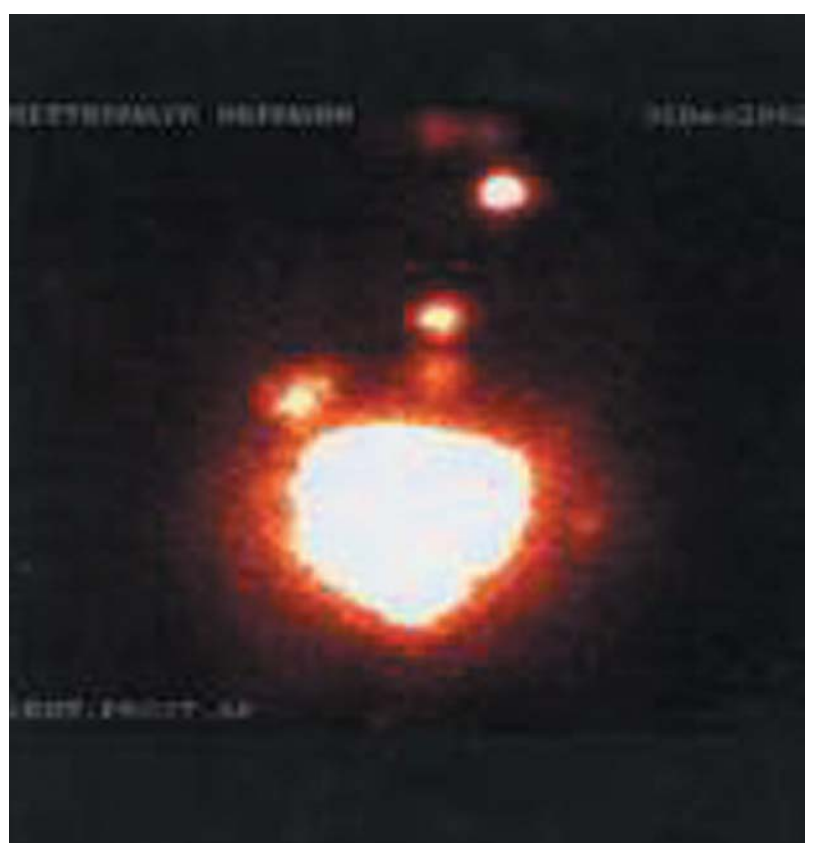

FIGURA 1. Imagen de gammacámara; 4 horas después de la inyección de coloide de Tecnecio $99 \mathrm{~m}$ se observan varios ganglios centinelas marcados por la radioactividad. 


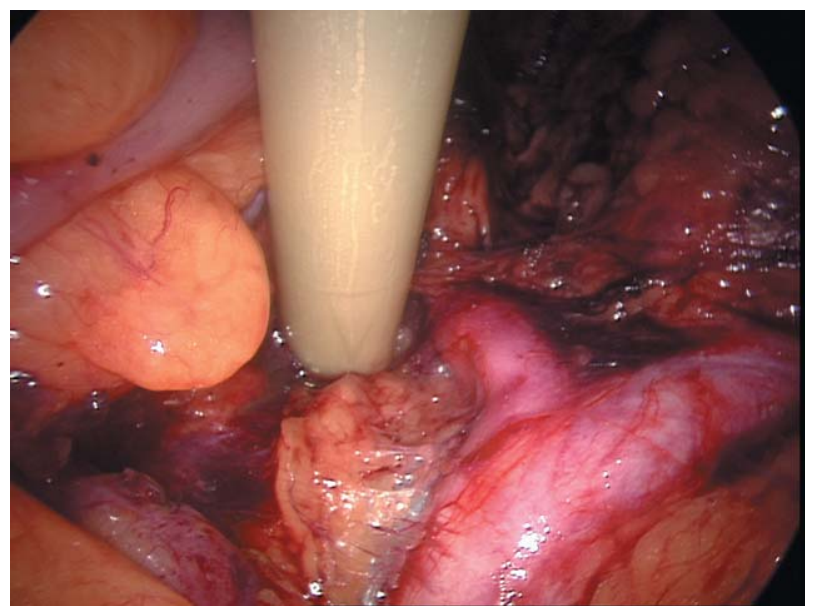

FIGURA 2. Sonda de gamma laparoscópica rastreando el área presacra derecha.

Para que la detección del GC se realice con éxito, son de especial importancia la estimación del tiempo de tránsito en el torrente linfático hasta el $\mathrm{GC}$ al igual que de la falta del paso del radiofármaco de forma significativa de la cadena ganglionar primaria a la secundaria. Estas características quedan determinadas por la estructura bioquímica y el tamaño molecular del vector. Actualmente, la albúmina humana y el coloide de azufre son los vectores que habitualmente se utilizan en la detección del GC.

En los estudios iniciales, se promulgó utilizar partículas de radiocoloides de tamaño relativamente pequeño para que penetrasen de una manera más rápida en los capilares linfáticos y así diseminarse ampliamente a través de toda la cadena linfática ${ }^{5}$. Sin embargo, en esa época la finalidad de la gammagrafía era visualizar la mayoría de los ganglios linfáticos para la caracterización de patrones de flujo linfático. Por el contrario, en la disección del GC el objetivo es conceptualmente diferente, es decir, la detección sólo del primer escalón linfático. Estudios efectuados en pacientes con cáncer de mama han demostrado que el uso de partículas de menor tamaño visualiza un mayor número de ganglios linfáticos, haciendo más difícil la disección del $\mathrm{GC}^{6}$. Consecuentemente, los coloides que se utilizan habitualmente en el estadiaje del GC tienen un tamaño de entre $100 \mathrm{~nm}-400 \mathrm{~nm}$.

La administración de radiofármacos debería estar basada en el conocimiento de los patrones de drenaje linfáticos del órgano estudiado. Tenemos que enfatizar dos puntos dentro del contexto del cáncer de próstata: primero, cada lóbulo prostático drena fundamentalmente en la cadena linfática ipsilateral; segundo, no hay diferencias en el drenaje linfático de las zonas transicional y la periférica ${ }^{7}$.

Para el estadiaje del GC se administran a través de un abordaje transrectal entre 1 y 3 inyecciones del radiofármaco seleccionado en cada lóbulo prostático. Basado en los estudios en modelo animal, Wawroschek et al., concluyen que para una mayor detección del GC, 3 inyecciones en cada lóbulo pueda tener ventajas sobre la técnica donde sólo se utiliza una inyección ${ }^{8}$. En nuestra práctica inyectamos mediante una aguja de $21 \mathrm{G}, 1 \mathrm{~mL}(100 \mathrm{MBq})$ de albúmina sérica humana marcada con Tc99m de forma bilateral en la zona periférica de la glándula prostática. Este procedimiento se realiza bajo sedación $24 \mathrm{~h}$ antes de la cirugía.

Tan sólo 2 pacientes de entre más de 150 casos, han experimentado una complicación séptica tras la administración intraprostática del coloide. Ambos paciente relataron una infección urinaria y una prostatitis aguda, respectivamente, varias semanas antes de que tuviera lugar la intervención.

Tras la inyección, alguno de los coloides no consiguen penetrar en los capilares linfáticos. Otros, sin embargo, especialmente aquellos de menor tamaño, pueden discurrir por el torrente linfático más allá del GC. Aún más, los radionúcleotidos libres entran en el torrente sanguíneo para ser captados por los diferentes órganos. De hecho, la captación de las moléculas transportadoras en el GC es tan sólo del 0,12\% del total de la dosis administrada. Por tanto, sólo los "hot spots” localizados en las áreas anatómicamente conocidas del drenaje linfático prostático deberían considerarse como posibles GC.

La gammagrafia preoperatoria aporta información sobre la cantidad y la localización del GC (Fig. 1). Con este fin, un colimador convencional de $10^{5}$ cuentas por imagen es el apropiado. Tras 3-4 h de la inyección del radionucleótido se obtienen imágenes estáticas planares tanto de los planos ventrales como dorsales. En el estudio de Wawroschek et al., el 78\% de GC se detectaron durante la cirugía con la gammagrafía planar ${ }^{9}$. 
Esta discrepancia entre la cirugía y técnica de imagen puede ser debida al relleno de la vejiga con el radiotrazador una vez filtrado por los riñones. Tal interferencia hace difícil conseguir una correcta visualización de las áreas hipogástricas y presacra. Pueden utilizarse cámaras de multidetección junto cortes seriados de la imagen para obtener una localización anatómica más precisa de los ganglios linfáticos ${ }^{10}$.

La mayoría de los estudios actuales abogan por un periodo de $24 \mathrm{~h}$ entre la inyección del radiofármaco y la cirugía. La radiación emitida por el radionúclido almacenado en el GC, es detectada intraoperatoriamente a través de la ventana de energía localizada en el extremo de la gamma sonda (Fig. 2). El sistema de detección traduce la radioactividad detectada en señales acústicas y digitales. Con el incremento del interés clínico, la tecnología de las sondas ha ido evolucionando rápidamente en los últimos años, fundamentalmente para optimizar su capacidad portátil, facilidad de empleo y detección de la señal emitida. Se ha diseñado una sonda especial para laparoscópia que tiene en cuenta sus limitaciones durante la manipulación y el tamaño de los trócares. Esta sonda tiene una ventana de energía lateral de $90^{\circ}$ cerca de la punta, la cual permite explorar las paredes laterales pélvicas con una alta resolución espacial. La detección intraoperatoria del GC y su exéresis puede realizarse mediante una abordaje abierto o laparoscópico ${ }^{4}$. El rango de detección intraoperatoria del GC varía del $87,5 \%$ al $100 \%$. Los principales factores que determinan el éxito de la detección del GC incluyen las características del radiofármaco usado, la técnica de administración y los límites de tiempo desde el marcaje del coloide a la cirugía. Las situaciones descritas que se han asociado con el fracaso en la identificación del GC son: cirugía prostática o pélvica previa y la enfermedad metastásica avanzada que haya causado una destrucción completa de la estructura ganglionar ${ }^{11}$. Esta última condición se debería detectar con las técnicas de imagen durante el preoperatorio. Se extraen todas las adenopatías que muestran radioactividad (Fig. 3). Si se detecta tumor en la sección congelada, se realiza una LNDP extendida bilateral (Fig. 4).

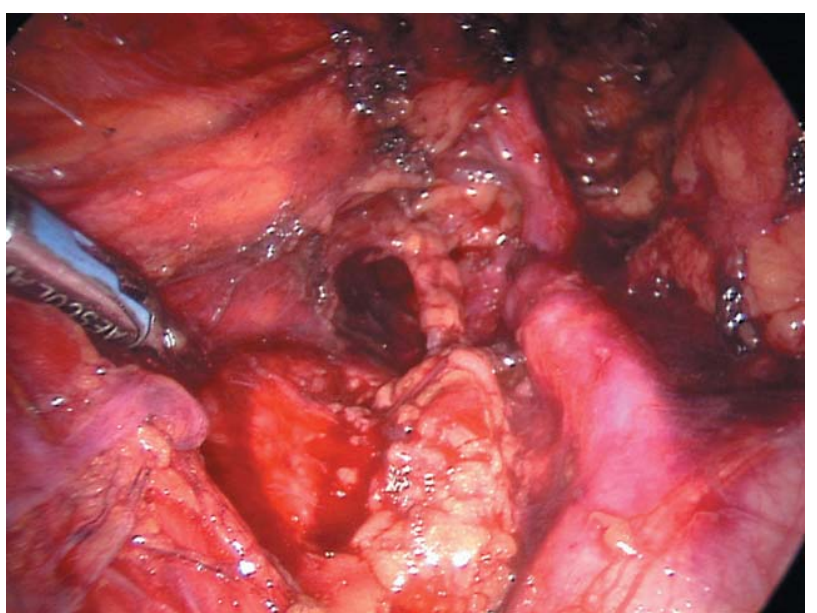

FIGURA 3. El paquete ganglionar presacro derecho ha sido identificado como ganglio centinela debido a su radioactividad y, por lo tanto es extirpado.

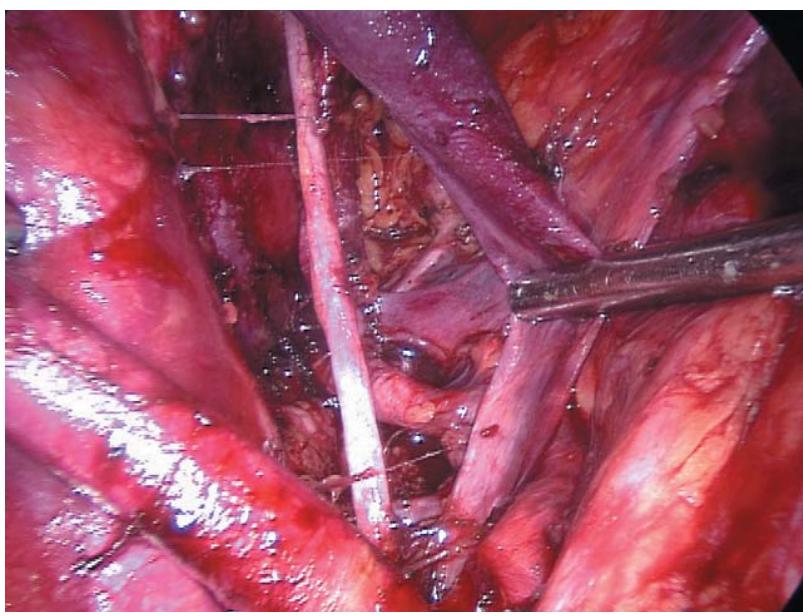

FIGURA 4. LDNP extendida izquierda: es necesaria si en el estudio por congelación, se encuentran metástasis en el ganglio centinela extirpado o si no hay radioactividad en la pared pelviana.

\section{RESULTADOS}

El grupo de Augsburg fue el primero en realizar la LDNP del GC radioguiada en el cáncer de próstata ${ }^{3}$. Este grupo tiene la mayor experiencia en la actualidad con más de 1.000 pacientes ${ }^{12}$. Han detectado una afectación ganglionar en el $19,6 \%$ de los pacientes, y un $63,3 \%$ de las metástasis estaban localizadas fuera del territorio de la LNDP limitada estándar. Se realizó LDNP extendida de forma complementaria a la LDNP del GC en 228 hombres con cáncer de próstata de alto riesgo ${ }^{13}$. De estos pacientes, 96 tuvieron metástasis ganglionares, y tan sólo en 2 no se detectaron mediante la LDNP del GC. 
26/96 pacientes $(27,1 \%)$ presentaron afectación metastásica en ganglios no centinelas lo que indica una diseminación a escalones linfáticos secundarios.

En nuestra primera serie, la tasa de detección de las metástasis ganglionares fue del 12,9\% en 71 pacientes con un PSA medio de $8,9 \mathrm{ng} / \mathrm{mL}^{4}$. El $72,7 \%$ de las adenopatías se localizaron fuera de la fosa obturatriz. Las metástasis ganglionares se encontraron exclusivamente en aquellas adenopatías marcadas con el trazador radiactivo. La metástasis más pequeña tuvo $0,2 \mathrm{~mm}$ de diámetro y se detectaron varias micrometástasis. El abordaje laparoscópico transperitoneal demostró estar indicado para la LDNP del GC y fue superior a la extraperitoneoscopia ya que la disección de los ganglios iliacos internos fue más precisa, y el riesgo de linfocele fue más bajo.

En otro estudio estudiamos la exactitud diagnóstica del 18 Fluor Cholin PET-CT y la LDNP laparoscópica del GC; los resultados fueron validados por medio de la LDNP laparoscópica extendida $^{11}$. La tasa de metástasis ganglionares en 20 pacientes fue del 50\%. La LDNP laparoscópica del GC demostró ser muy eficiente para la detección de micrometástasis. Cholin PET-CT tiene una alta especificidad, pero su sensibilidad es claramente más baja que la LDNP del GC. La LDNP extendida se considera el "gold standard", pero determinadas metástasis pueden no detectarse con este método y sí mediante LDNP del GC solo. El tamaño tumoral mínimo que pueda detectarse mediante PET$\mathrm{CT}$ tiene que investigarse en mayores series pero parece estar en el rango de $8 \mathrm{~mm}$.

En nuestra publicación más reciente, se presenta los datos de 140 pacientes diagnosticados de CPO entre noviembre de 2001 a enero 2005, en los que se realizó LDNP laparoscópica del GC previa a la PTR, también laparoscópica ${ }^{14}$. En la gammagrafia preoperatoria, se detectaron GC de forma bilateral en 113 pacientes (80,7\%), de forma unilateral en $20(14,2 \%)$ y no se detectaron en $6(4,2 \%)$, mientras que en la realizada durante la cirugía se detectaron en 96 pacientes (68,6\%), $36(25,7 \%), 8(5,7 \%)$ respectivamente. Los GC se hallaron en la misma localización con ambos procedimientos en 99 de 140 pacientes $(70,7 \%)$. Al menos se objetivó un GC en 133 pacientes $(95,3 \%)$.
La disección del GC se completó en todos los casos sin necesidad de conversión a cirugía abierta. El tiempo medio de cirugía fue de 51 minutos (28-97). Se obtuvieron un total de 540 GC con una mediana de 4 por paciente (1-20). La correlación entre la localización del GC preoperatoria e intraoperatoria mediante gammagrafia y metástasis ganglionares fue del 79\% (15/19) y 90\% (17/19) respectivamente. El 55,7\% (127/ 228) de estos GC se localizaron fuera de la fosa obturatriz. El examen histopatológico mostró metástasis en 19 pacientes $(13,5 \%)$ y 6 pacientes $(4,3 \%)$ tuvieron afectación bilateral. La incidencia en los grupos de riesgo bajo, intermedio y alto fue de $8,2 \%(6 / 73), 10 \%(5 / 50)$ y $47 \%(8 / 17)$ respectivamente. La mediana de PSA preoperatorio en estos pacientes fue de $10,8 \mathrm{ng} / \mathrm{mL}$ (SD 9,7). EL $71,4 \%$ de las metástasis $(20 / 28)$ se localizaron fuera de la fosa obturatriz. En cuanto a los grupos de riesgo bajo e intermedio, 66,6\% (4/6) y $60 \%(3 / 5)$ de los GC positivos, se detectaron fuera de dicha fosa, respectivamente. El tamaño medio de las metástasis en el GC fue de $2,3 \mathrm{~mm}$ (DE 1,7) y 10 de estas metástasis eran menores de $2 \mathrm{~mm}$, es decir micrometástasis. En 3 pacientes con afectación ganglionar no se realizó LDNP extendida, ya que la sección de las muestra congelada fue negativa. Además, en un paciente que tenía una gran cicatriz tras una amputación rectal (incluida LDN) por una neoplasia de recto, la LDNP extendida no se completó en un de los lados. En 17 de los 19 pacientes (85\%) con afectación ganglionar, las metástasis fueron detectadas exclusivamente en aquellas adenopatías obtenidas según la técnica del GC. 69,2\% (18/26) de los ganglios positivos eran únicos en las paredes pélvicas laterales. En un paciente se determinó la presencia de otra adenopatía positiva adicional no detectada con la técnica del GC (alrededor de la arteria ilíaca externa). En otro caso con metástasis no se observó ninguna actividad del trazador en las paredes laterales de la pelvis. La ausencia de detección de dicha actividad se constató en nuestro estudio en 52 de 280 pacientes (19\%). En 2 pacientes hubo que diferir la disección del GC porque desarrollaron una prostatitis tras la inyección del radioisótopo. A estos pacientes se le practicó una LDNP convencional por lo que fueron excluidos del estudio. 
La sonda de detección laparoscópica no se pudo utilizar por mal funcionamiento en un caso, indicándose por tanto una LDNP extendida y medición de la radioactividad in vitro. En 49 pacientes la disección del GC se siguió de LDNP extendida por ser positiva la pieza extemporánea (16 pacientes) y resultados inconclusos (33 pacientes) (Fig 4). 6 de estos 49 pacientes (12\%) presentaron complicaciones relacionadas con la intervención: una lesión de la vena ilíaca externa, una neuropraxia temporal (n. isquiático), una trombosis venosa profunda y 3 linfoceles. En los 91 pacientes en los que solamente se llevó a cabo la disección del GC, la tasa de complicaciones fue del $1 \%$ (lesión de la vena ilíaca externa). Todas las lesiones vasculares fueron identificadas y tratadas laparoscópicamente durante el mismo acto quirúrgico. Los 3 casos de linfocele ocurrieron tras abordaje transperitoneal y se drenaron percutáneamente y posterior marsupialización laparoscópica. No se ha descrito ninguna muerte durante la cirugía.

\section{DISCUSIÓN}

La LDNP del GC es una alternativa válida a la LDNP extendida. El número de ganglios positivos en nuestra experiencia se corresponde con la de otros grupos que realizan ambos procedimien$\operatorname{tos}^{2,3,12,13}$. Cuando la literatura actual estima la incidencia de adenopatías positivas entre el 2$7 \%$, se basan en trabajos en los que se ha utilizado LDNP limitada ${ }^{15,16}$. Los nomogramas validados de los que disponemos para la predicción de la afectación ganglionar, están basados en estadiaje clínico, Gleason y PSA preoperatorio ${ }^{17}$. Sin embargo, estos nomogramas predicen la probabilidad de que la fosa obturatriz esté afecta y no la incidencia real de diseminación ganglionar. Por otro lado, se ha cuestionado la necesidad de realizar LDNP rutinaria en los casos de enfermedad órgano-confinada debido en parte a la baja tasa de ganglios positivos ${ }^{18}$. No obstante, estos estudios están basados en la LDNP limitada, por lo que tienden a infravalorar la incidencia real de enfermedad avanzada e inducir a conclusiones erróneas respecto a la exactitud del estadiaje y manejo terapéutico.

Como consecuencia de que sólo se extirpan aquellos ganglios con riesgo de metástasis, la
LDN del GC es un procedimiento más fácil, más rápido y con menos tasa de complicaciones que la LDNP. Sin embargo, existen ciertos inconvenientes de esta técnica que deben de tenerse en cuenta para obtener unos resultados óptimos. El tamaño del coloide utilizado es de gran importancia, y determinará si aparte del primer escalón linfático, un secundario o terciario también se representa en la gammagrafia. Otro de los problemas es la no-visualización de los GC por la falta de radioactividad en las paredes pélvicas laterales. En estos casos la LDNP extendida se debe llevar a cabo. Esta falta de actividad detectada por el escáner durante la cirugía se produjo en 36 de nuestros pacientes $(25,7 \%)$ de forma unilateral y en $8(5,7 \%)$ de forma bilateral.

Las circunstancias que en la literatura están asociadas al fracaso de la identificación del GC son: cirugía pélvica o prostática previa y la completa destrucción de la estructura linfática por enfermedad metastásica avanzada ${ }^{11}$. Si el tejido linfático es reemplazado completamente por tumor, este ganglio queda bloqueado al torrente linfático y pasará a quedar marcado un segundo escalón. Este fenómeno se conocía ya por la experiencia con la linfografía. No obstante, el tamaño de estos nódulos tendría que ser lo suficientemente grande por lo que se detectarian mediante un TC preoperatorio ${ }^{11}$.

La LDNP extendida bilateral se tiene que realizar en todos los pacientes en los cuales se ha objetivado una afectación metastásica del GC. La sección de la pieza puede ser un falso negativo en un porcentaje alto. Como consecuencia, cierta diseminación linfática pasará desapercibida puesto que no se realizaría la LDNP extendida correspondiente. Esta situación ocurre en cerca del $40 \%$ de nuestros pacientes con ganglios positivos. Sin embargo, un análisis más detallado reveló que todos estos pacientes tuvieron micrometástasis por lo que la diseminación tumoral más allá del territorio primario fue improbable.

\section{CONCLUSIONES}

Cuando la LDNP está indicada, esta no debería limitarse a la exéresis de adenopatías según la técnica de LDNP limitada o estándar, sino que tiene que realizarse siguiendo el patrón de la LDNP extendida. Hay poca experiencia con la 
LDNP del GC, pero todos los datos publicados hasta ahora indican que este procedimiento pueda ser una alternativa a la LDNP extendida, ya que permite reducir la extensión de la LDNP sin comprometer la exactitud diagnóstica.

\section{REFERENCIAS}

1. Raghavaiah NV, Jordan WP. Prostatic lymphography. J Urol. 1979 Feb;121(2):178-181.

2. Bader P, Burkhard FC, Markwalder R, Studer UE. Is a limited lymph node dissection an adequate staging procedure for prostate cancer. J Urol. 2002 Aug; 168(2):514-8; discussion 518 .

3. Wawroschek F, Vogt H, Weckermann D, Wagner T, Hamm M, Harzmann R. Radioisotope guided pelvic lymph node dissection for prostate cancer. J Urol. 2001 Nov; 166(5):17151716.

4. Jeschke S, Nambirajan T, Leeb K, Ziegerhofer J, Sega W, Janetschek G. Detection of early lymph node metastases in prostate cancer by laparoscopic radioisotope guided sentinel lymph node dissection. J Urol. 2005 Jun;173(6):19431946.

5. Strand SE, Persson BR. Quantitative lymphoscintigraphy: Basic concepts for optimal uptake of radiocolloids in the parasternal lymph nodes of rabbits. J Nucl Med. 1979 Oct;20(10): 1038-1046.

6. Paganelli G, De Cicco C, Cremonesi M, Prisco G, Calza P, Luini A, Zucali P, Veronesi U. Optimized sentinel node scintigraphy in breast cancer. Q J Nucl Med. 1998 Mar;42(1): 49-53.

7. Brössner C, Ringhofer H, Hernady T, Kuber W, Madersbacher S, Pycha A. Lymphatic drainage of prostatic transition and peripheral zones visualized on a three-dimensional workstation. Urology. $2001 \mathrm{Feb}$;57(2):389-393.

8. Wawroschek F, Wengenmair H, Senekowitsch-Schmidtke R, Hamm M, Henke J, et al. Prostate lymphoscintigraphy for sentinel lymph node identification in canines: reproducibility, uptake, and biokinetics depending on different injection strategies. Urol Res. 2003 Jul;31(3):152-158. Epub 2003 Mar 25.

9. Wawroschek F, Vogt H, Wengenmair H, Weckermann D, Hamm M, Keil M et al. Prostate lymphoscintigraphy and radio-guided surgery for sentinel lymph node identification in prostate cancer. Technique and results of the first 350 cases. Urol Int. 2003;70(4):303-310.
10. Corvin S, Schilling D, Eichhorn K, Hundt I, Hennenlotter $\mathrm{J}$, Anastasiadis AG, et al. Laparoscopic sentinel lymph node dissection - a novel technique for the staging of prostate cancer. Eur Urol. 2006 Feb;49(2):280-285. Epub 2005 Dec 9.

11. Haecker A., Jeschke S., Leeb K., Prammer K., Ziegerhofer J., Sega W., Langsteger W., Janetschek G: Detection of pelvic lymph node metastases in patients with clinically localized prostate cancer: comparison of $\mathrm{FCH} \mathrm{PET} / \mathrm{CT}$ and laparoscopic radioisotope-guided sentinel lymph node dissection. In publishing process, J Urol 2006.

12. Weckermann D, Dorn R, Trefz M, Wagner T, Wawroschek F, Harzmann R: Sentinel lymph node dissection for prostate cancer: experience with more than 1.000 patients. J Urol. 2007 Mar; 177(3):916-920.

13. Weckermann D, Dorn R, Holl G, Wagner T, Harzmann R. Limitations of radioguided surgery in high-risk prostate cancer. Eur Urol. 2007 Jun;51(6):1549-56; discussion 1556-8. Epub 2006 Sep 11 (Epub ahead of print)

14. Jeschke S, Beri A, Grüll M, Ziegerhofer J, Prammer P, Leeb $\mathrm{K}$, et al. Laparoscopic radioisotope guided sentinel lymph node dissection in staging of prostate cancer. Eur Urol. 2007 Mar 28.

15. Pound CR, Partin AW, Epstein JI, Walsh PC. Prostate-specific antigen after anatomic radical retropubic prostatectomy. Urol Clin North Am. 1997 May;24(2):395-406

16. Catalona WJ, Smith DS: Cancer recurrence and survival rates after anatomic radical retropubic prostatectomy for prostate cancer: intermediate-term results. J Urol. 1998 Dec; 160(6 Pt 2):2428-2434.

17. Partin AW, Kattan MW, Subong EN, Walsh PC, Wojno KJ, Oesterling JE, et al. Combination of prostate-specific antigen, clinical stage, and Gleason score to predict pathological stage of localized prostate cancer. JAMA. 1997 May 14; 277(18):1445-1451

18. Bishoff JT, Reyes A, Thompson IM, Harris MJ, St Clair SR, Gomella L, Butzin CA. Pelvic lymphadenectomy can be omitted in selected patients with carcinoma of the prostate: development of a system of patient selection. Urology. 1995 Feb;45(2):270-274.

Correspondencia autor: Dr. G. Janetschek

Professor of Urology. Elisabethinen Hospital Dept of Urology. Fadingerstr. 1. 4020 - Linz. Austria Tel.: +4370 76764551

E-mail: guenter.janetschek@elisabethinen.or.at Información artículo. Original 